\title{
La "Investigación" en los cursos de licenciatura de la modalidad a distancia: actualidad y futuro en el ejemplo de la UFMA
}

\author{
Reinaldo Portal Domingo*
}

\section{Resumo}

O presente artigo apresenta um grupo de reflexões sobre a disciplina Metodologia Científica nos cursos de licenciatura na modalidade em EAD na UFMA, em particular: o conteúdo da mesma, os materiais didáticos para seu desenvolvimento, as atividades e tarefas de pesquisa a serem desenvolvidas dentro dos cursos, assim como o papel da ferramenta pesquisa como eixo transversal que deve ser assimilada pelos alunos durante todas as disciplinas do curso, para lograr uma ampla preparação nessa área, incorporar a ferramenta no dia a dia de sua formação docente, preparando o aluno para enfrentar com êxito seu TCC, além de incorporar a "pesquisa" para seu futuro profissional.

Palavras-chaves: metodologia científica; educação a distância; atividades de investigação.

\section{The research in licentiate courses in the distance learning modality: the present and the future in the example of UFMA}

\section{Abstract}

The present article shows a group of reflections about the discipline Science Methodology in licentiate degree courses in the modality in distance learning in UFMA, in particular: the content of the discipline, the educational materials for its development, the activities and research tasks to be developed within the courses, as well as the role of the research tool as a transversal axis that must be assimilated by the students throughout all

Universidad Federal de Maranhão (UFMA).rportaldomingo@yahoo.com.br 
the disciplines of the course, to achieve a broad preparation in this area, to incorporate the tool in the day to day of its teacher training, preparing the student to successfully face their Term Paper, in addition to incorporating "research" into their professional future.

Keywords: scientific methodology; distance education; research activities.

\section{Introducción}

Hoy la educación a distancia (EAD) en Brasil es un fenómeno en crecimiento que vino para quedarse y que cada día se desarrolla más, con la inclusión de diferentes tecnologías y metodologías que hacen de la EAD una modalidad educativa de amplia aceptación de jóvenes y adultos de todo el país. Basta decir que 7,3 millones de alumnos que tienen la educación superior brasileña, el 15\% se corresponde a los alumnos de la EAD (Portal Brasil, Censo 2013). Por las “aulas” de esta modalidad han pasado un aproximado de 3 millones de alumnos en los últimos 5 años y su número sigue en aumento.

En el proceso de desarrollo de la EAD en Brasil, son diversos los obstáculos encontrados. Algunos de ellos, de carácter externo, mayoritariamente relacionados al incremento de recursos financieros y la aplicación de políticas públicas correctas, y otros de carácter interno, relacionados a los métodos y procedimientos de como ofrecer este tipo de educación en el país. Dentro de esas dificultades, la metodología de investigación y su didáctica es uno de los temas menos discutidos en la actualidad. A manera de ejemplo podemos citar, que en los últimos congresos internacionales de EAD (2012, 2013, 2014, 2016 y 2017) de la Asociación Brasileña de Educación a Distancia $(\mathrm{ABED})$, los trabajos relacionados con esta área son minoritarios. No obstante, observamos un interés creciente de un grupo de especialistas que vienen trabajando en este tema. Como ejemplo podemos mostrar las dos mesas redondas realizadas en los congresos de la ABED (2016 y 2017) coordinadas por este autor para tratar el tema de la investigación en los cursos de EAD del país.

Es bueno también resaltar el trabajo, que bajo la conducción de la especialista Vani Moreira Kenski, se viene realizando por la ABED en los últimos años sobre "los grupos que investigan EAD en Brasil" como un 
esfuerzo en saber que se investiga sobre esta temática por las universidades nacionales (CIEAD, 2017).

La investigación pasa a ser un importante tema para docentes y discentes, porque constituye el núcleo central del aprendizaje, proceso que se realiza tanto en los cursos de graduación presencial como a distancia. Por esa razón trabajar en función de desarrollar las habilidades investigativas dentro de todos los cursos debe ser objetivo importante de las universidades en el siglo XXI.

Uno de los principales elementos que la universidad contemporánea debe desarrollar en los alumnos es el componente investigación como herramienta de trabajo para poder enfrentar con éxito los diferentes desafíos de toda su etapa de formación y preparación para el mundo del trabajo.

Actualmente vemos con preocupación que los contenidos y actividades de investigación dentro de los cursos a distancia en la Universidad Federal de Maranhão (UFMA) se reducen al contenido de la asignatura Metodología Científica con una duración aproximada entre 60-90 horas clases y a la elaboración de la monografía de conclusión de estudios. La investigación como herramienta docente debe estar presente durante la realización de todo el curso, debe pasar a ser una estrategia de trabajo, ya que parafraseando a Pedro Demo, quien investiga bien, aprende bien. Es misión de la EAD garantizar que los alumnos aprendan bien, e investigar es uno de los caminos principales para acompañar y apoyar el proceso docente de inicio a fin. La investigación debe transformarse en estilo de trabajo científico de todos los alumnos, no importa la modalidad en que estudien. Debemos lograr que lo que el alumno aprenda en el área de metodología científica sea aquello que aplique durante el período de estudio de la educación superior, $\mathrm{y}$ lo incorpore a su futura vida profesional.

Una reflexión interesante seria poder responder a las siguientes interrogantes: ¿Cuáles son los elementos de investigación que no deberían faltar en la asignatura Metodología Científica de los cursos de licenciatura a distancia? ¿Con qué recursos didácticos cuenta el profesor de esta asignatura a distancia para realizar la mediación pedagógica dentro de la asignatura? Qué actividades de investigación los alumnos de la EAD deben realizar durante todo el período de su formación? 
Fue tarea de este trabajo de pesquisa buscar sustento teórico/práctico para intentar responder a las interrogantes antes mencionadas para apoyar el desarrollo de la metodología científica y la herramienta "investigación" de los cursos de licenciatura en la modalidad a distancia.

\section{Desarrollo}

El tema relacionado con la investigación y su papel en la docencia es hoy objeto de debate permanente dentro del escenario nacional en Brasil (SILVA, 1990; MARQUES, 1997; GALIAZZI; MORAES, 2002; BAGNO, 2009; BRANDÃO, 2003; MORAES; LIMA, 2002; PRESTES, 2003; DEMO, 2000a, 2001, 2002, 2004a, 2004b, 2006, 2008a, 2008b, 2015) en España (BISQUERRA, 2004; GARCÍA-LLAMAS, 2003; PÉREZ JUSTE, 2006, 2011, 2012; GALÁN, 2003, 2013; RIO, 2005, 2008) y en Cuba (COLECTIVO DE AUTORES, 2003; RUIZ, 2007; CEREZAL; FIALLO, 2004; entre otros).

Saber investigar se traduce en la actualidad en aplicar los conocimientos científicos a todas las esferas de la vida, es colocar la investigación como filosofía de vida y resolver los problemas cotidianos aplicando las herramientas que hoy se tienen en el campo de la investigación. Para ello los recursos con que se cuenta hoy en la Web 2.0 son innumerables y es responsabilidad de las universidades capacitar a los docentes y enseñar a los alumnos a utilizar estas herramientas en función del proceso de enseñanza-aprendizaje.

Según el especialista brasileño en metodología de la investigación Pedro Demo (2008, traducción del autor), "Es muy posible aprender bien con apoyo de las nuevas tecnologías, siendo idea crucial la de saber investigar y elaborar. El desafío es transformar las plataformas virtuales en plataformas de investigación".

Una de las tareas realizadas es la definición de los elementos fundamentales (núcleos básicos de contenido) de la asignatura Metodología Científica y de los materiales didácticos digitales que necesitarán en la asignatura. Para ello nos basamos en la clasificación de Pedro Demo en lo relacionado con la Calidad formal de la investigación: 


\begin{tabular}{|c|c|}
\hline $\begin{array}{l}\text { Metodología } \\
\text { científica }\end{array}$ & $\begin{array}{l}\text { Cuestionamiento del método científico y demarcación científica; crite- } \\
\text { rios formales y políticos de la cientificidad; estudio de los paradigmas en } \\
\text { uso (positivismo, dialécticas, estructuralismo, empirismos); discusión de } \\
\text { propuestas más cuantitativas y más cualitativas (investigación participati- } \\
\text { va, investigación-acción, evaluación cualitativa; mezclas); neutralidad-ob- } \\
\text { jetividad; saber pensar e intervenir (DEMO, 1994, 1995, 2000). }\end{array}$ \\
\hline Métodos y técnicas & $\begin{array}{l}\text { Producir, usar e interpretar datos empíricos; procedimientos de investi- } \\
\text { gación (cuestionarios abiertos y cerrados; observación participante; his- } \\
\text { torias de vida y orales; grupos focales); fundamentos estadísticos de la } \\
\text { calidad de los datos; las muestras; análisis de regresión, de correlación, } \\
\text { de componentes principales, factoriales (DEMO, 2002c, SCHRADES, } \\
\text { 2002; VIA; DENCKER, 2001). }\end{array}$ \\
\hline Dominio teórico & $\begin{array}{l}\text { Conocimiento/discusión de teorías relevantes; saber usar estas, descons- } \\
\text { truir/reconstruir; producción de textos propios; lecturas y contra lectu- } \\
\text { ras; autores centrales, clásicos, nuevos espacios teóricos,; paradigmas en } \\
\text { uso y nuevos paradigmas (DEMO, 1999a, 1994; MORIN, 2002). }\end{array}$ \\
\hline Dominio técnico & $\begin{array}{l}\text { Manejo crítico y creativo de las técnicas incluidas en los espacios profe- } \\
\text { sionales (p. ej. Técnicas de gerenciamiento de empresas; técnicas peda- } \\
\text { gógicas y de alfabetización; técnicas de uso de preservación del suelo) } \\
\text { (DEMO, 2000a). }\end{array}$ \\
\hline $\begin{array}{l}\text { Inter, transasignatura- } \\
\text { riedad }\end{array}$ & $\begin{array}{l}\text { Superación de la asignaturaridad artificial de los cursos; cuidar de la es- } \\
\text { pecialización verticalizada (necesaria para cualquier profundización del } \\
\text { conocimiento) sin incidir en el "idiota especializado"; no perderse en la } \\
\text { horizontalidad para no convertirse en "especialista en generalidades"; } \\
\text { trabajar en equipo (consorcio de especialistas que saben aprender recí- } \\
\text { procamente) (MORIN, 1995; DEMO, 2000). }\end{array}$ \\
\hline $\begin{array}{l}\text { Nuevas } \\
\text { tecnologías }\end{array}$ & $\begin{array}{l}\text { Alfabetizaciones (digital, tecnológica, informática...); producción y uso } \\
\text { de nuevas lenguajes virtuales (animadas, internet, 3D, audio, vídeo, jue- } \\
\text { gos electrónicos); internet como manantial infinito de investigación y } \\
\text { elaboración, no del plagio; habilidades comunicativas virtuales, grupos } \\
\text { de investigación virtual; espacios alternativos de aprendizaje virtual; pre- } \\
\text { sencia virtual (DEMO, 2007); }\end{array}$ \\
\hline Propedéutica básica & $\begin{array}{l}\text { Algunos fundamentos del saber pensar: filosofía (crítica de la ciencia), } \\
\text { lenguaje (saber comunicarse, relacionarse), matemáticas (lenguaje numé- } \\
\text { rica y digital); manejo del inglés como lengua franca, en especial tecnoló- } \\
\text { gica; saber estudiar, argumentar, preguntar, en especial el auto indagación } \\
\text { (DEMO, 1996a); }\end{array}$ \\
\hline Evaluar/analizar & $\begin{array}{l}\text { Capacidad de diagnóstico a partir de su área profesional; capacidad de } \\
\text { análisis de la producción científica en el área; visión de corto, medio y } \\
\text { principalmente de largo alcance; percepción de los cambios, innovación, } \\
\text { cambios de paradigmas, paradigmas viejos, nuevos y previsión del co- } \\
\text { nocimiento; saber dar cuenta de la realidad de modo flexible (DEMO, } \\
2006 \text { a). }\end{array}$ \\
\hline
\end{tabular}


Según Pedro Demo, "actividades de investigación" son aquellas encaminadas a incluir en la vida del alumno el compromiso de estudiar, investigar, elaborar, argumentar. Este autor clasifica esas actividades de la siguiente forma:

\begin{tabular}{|c|c|c|}
\hline En tono mayor & $\begin{array}{l}\text { 2) } \\
\text { 3) } \\
\text { 4) } \\
\text { 5) }\end{array}$ & $\begin{array}{l}\text { Realización de proyectos de investigación (difícil de rea- } \\
\text { lizar por el alumno, aunque viable, hasta cierto punto, en } \\
\text { especial cuando está insertado en el proyecto de algún } \\
\text { profesor); } \\
\text { Elaboración de un proyecto propio de investigación (in- } \\
\text { cluyendo tal vez algunas de las etapas de realización); } \\
\text { Elaboración de texto científico (para ser defendido en se- } \\
\text { minario pre-establecido) siguiendo las normas de cientifi- } \\
\text { cidad para estos casos; } \\
\text { Elaboración de trabajos de conclusión de curso o de eta- } \\
\text { pas semestrales; } \\
\text { Investigaciones más trabajadas en ambientes virtuales, in- } \\
\text { dividuales o en grupos. }\end{array}$ \\
\hline En tono medio & $\begin{array}{l}\text { 1) } \\
\text { 2) } \\
\text { 3) } \\
\text { 4) } \\
\text { 5) }\end{array}$ & $\begin{array}{l}\text { Estudio más exhaustivo de teorías y autores, con la elabo- } \\
\text { ración correspondiente; } \\
\text { Desconstrucción/reconstrucción de prácticas pertinentes; } \\
\text { Seminarios que suponen la elaboración individual de los } \\
\text { participantes; } \\
\text { Grupos permanentes de estudio, también virtuales, con } \\
\text { producción consecuente y sistemática de conocimientos; } \\
\text { Investigaciones de medio porte virtual, individual o en } \\
\text { grupos. }\end{array}$ \\
\hline En tono menor & $\begin{array}{l}\text { 1) } \\
\text { 2) } \\
\text { 3) } \\
\text { 4) } \\
\text { 5) } \\
\text { 6) } \\
\text { 7) } \\
\text { 8) } \\
\text { 9) } \\
\text { 10) }\end{array}$ & $\begin{array}{l}\text { Interpretación crítica de autores y teorías, en forma de re- } \\
\text { sumen, reseña (no copia); } \\
\text { Estudio individual o en grupo de ciertas categorías, teo- } \\
\text { rías, autores, teniendo como resultado pequeños textos; } \\
\text { Discusión de polémicas pertinentes, con base en la autori- } \\
\text { dad del argumento; } \\
\text { Búsquedas en la internet de argumentos relevantes para } \\
\text { cierta cuestión; } \\
\text { Ejercicios académicos de carácter introductorio, inducien- } \\
\text { do a la elaboración propia; } \\
\text { Textos preliminares de autoría incipiente; } \\
\text { Dar cuenta de un libro o de un autor con visible descons- } \\
\text { trucción y reconstrucción; } \\
\text { Desarrollo de argumentos específicos para situaciones es- } \\
\text { pecíficas, por ejemplo, en el contexto de un debate; } \\
\text { Ejercicios metodológicos; } \\
\text { Aprovechamiento de información, datos, ambientes vir- } \\
\text { tuales, de modo reconstructivo. }\end{array}$ \\
\hline
\end{tabular}


Falta definir como colocar estas actividades dentro de las asignaturas de los cursos de la modalidad a distancia. Este aspecto parece ser una tarea nada fácil de poder combinar con todos los profesores para trabajar "la investigación" como un eje transversal.

En la actualidad todas las universidades están llamadas a producir ciencia y preparar a sus alumnos para adentrarse en el mundo de la ciencia. Esto significa que las universidades deben trabajar para desarrollar en sus alumnos aquellos componentes teórico-prácticos necesarios y suficientes que lo coloquen a la altura de las exigencias científico-tecnológicas del siglo XXI, lo que es lo mismo, tener una formación general que les permita entrar en el mundo de la producción de conocimientos y las innovaciones, elementos claves de la actual etapa de desarrollo del mundo contemporáneo. La ciencia fue, es y será el motor impulsor del desarrollo. De ahí la gran responsabilidad que tienen los sistemas de educación y en particular la universidad y los cursos a distancia en la preparación de ese hombre nuevo que debe actuar y pensar con método científico.

Resulta interesante el artículo de la especialista Clara Pereira Coutinho (2012), Investigar on-line: desafios y oportunidades, en que nos muestra como hoy en día la Internet se ha convertido en una fuente inacabable de recursos para la investigación, pero sabemos que una cosa es investigar para obtener un resultado, donde las herramientas de la Web 2.0 son de gran importancia en la actualidad y otra cosa es el aprender a investigar como proceso y más aún en la modalidad a distancia, que es el objetivo fundamental de nuestro trabajo.

Los conocimientos sobre ciencia y metodología de la investigación no son exclusivos de las universidades. Se sobre entiende que nuestros alumnos ya desde su período de formación en la escuela básica tienen que comenzar a trabajar con estos elementos. Lamentablemente los alumnos que ingresan actualmente en las universidades poseen pocos conocimientos en el campo de la investigación, por lo que la tarea de aprender a investigar se inicia de forma seria a partir de la universidad, concretándose aún más en los cursos de post-graduación, latto sensu (especialización) y strictu senso (maestría y doctorado).

La opinión generalizada de docentes e investigadores del área es que las universidades no están cumpliendo cabalmente con su papel de 
inclusión de sus alumnos en el mundo de la investigación, independientemente de la modalidad educativa si presencial o a distancia. Ejemplo de esto es la baja participación de los alumnos en los grupos de investigación existentes, así como la realización sistemática de tareas de investigación. Refiriéndose al Programa de Becas para la Iniciación Científica de alumnos en Brasil, conocida por las siglas PBIC, el especialista Pedro Demo ha expresado, que los alumnos que participan de estos programas e investigan bien, aprenden bien. Solo que el PBIC en Brasil abarca a un limitado grupo de alumnos, sobre todo a aquellos que tienen un marcado interés por la investigación y motivados por adquirir una beca.

Es importante destacar que para la gran mayoría de los alumnos de la EAD no existe la posibilidad de participar en grupos de pesquisa y el PBIC está establecido para alumnos de los cursos presenciales.

Precisamente una de nuestras propuestas es establecer "grupos de pesquisa" en los Polos de Apoyo Institucional de Educación a Distancia (PAIED), para incentivar aquellos alumnos que se interesan por la investigación y poder desarrollar esta importante herramienta de trabajo.

A diferencia de los cursos presenciales de la UFMA, en los cursos de la modalidad EAD no existen profesores de asignaturas con grupos de alumnos para investigar, no existen grupos de investigación formalmente organizados y aprobados, no existen Becas de Iniciación Científica (PBIC) para incentivar el trabajo de alumnos de la graduación para participar junto con profesores y otros especialistas en grupos de investigación por áreas o líneas de investigación.

Entonces, queda claro que debemos empezar a trabajar en la organización de actividades de pesquisa que tributen a la preparación de nuestros alumnos en el campo de la investigación de inicio al fin de los cursos con el objetivo de llegar al momento del TCC con suficientes conocimientos y herramientas de investigación que permitan a nuestros alumnos la culminación y defensa de los TCC con alto nivel de preparación.

Por eso estamos proponiendo la creación de grupos de pesquisa en nuestros PAIED. Para conocer com más detalles sobre este trabajo, los interesados deberán entrar en contacto con la Coordinación Pedagógica del NEAD/UFMA en: < http://portais.ufma.br/PortalUnidade/nead/>. 
Cada grupo debe tener un líder, o sea un profesor responsable de la organización y conducción del grupo, que debe ser seleccionado entre los especialistas del Polo que quieran enfrentar la tarea y que tengan un perfil relacionado con la investigación como herramienta de trabajo. Como sugerencia proponemos poner en manos del asesor a docencia del polo (figura nueva creada por la CAPES para los cursos de la UAB) esta responsabilidad, a partir de su vínculo con los elementos pedagógicos de la formación de docentes, aunque pudiera ser cualquier otro especialista del polo que se identifique y comprometa con la realización de esta tarea. La Coordinación Pedagógica del NEAD centrará la tarea de proponer las actividades relacionadas con "pesquisa" que serán realizadas en cada PAIED.

Las posibilidades de acceder a la investigación científica se encuentra inalcanzable para muchos alumnos de la educación superior de Brasil y específicamente para los alumnos de la EAD, que tienen, además de las limitaciones descritas anteriormente, por muchos estudiosos de este tipo de educación, tales como: distancia del campus (o polo presencial), dificultades de acceso a una bibliografía más amplia por falta de bibliotecas bien estructuradas, la carencia de tiempo para dedicarse a la investigación, algo bien común en alumnos adultos y trabajadores, característico de esta modalidad, Internet de banda ancha y con buena calidad para un acceso a la Web 2.0, unido a las carencias de una preparación previa de la educación básica recibida en muchos casos en ciudades del interior del país y limitadas habilidades en el uso de las TIC, ha perjudicado grandemente la producción científica, lo que repercute seriamente en la utilización de la investigación como herramienta de trabajo en diferentes actividades académicas y en la realización de los trabajos de culminación de estudios, lo que en muchos casos se convierte en el único momento real de contacto del alumno con la investigación.

Los especialistas L. C. Oliveira y H. R. Oliveira Júnior (2012), en su artículo La pesquisa en la educación a distancia: un análisis acerca de la asignatura Metodología de la Pesquisa en el curso de Administración de la UFMA, presentan un grupo de problemas existentes en el proceso de elaboración de los trabajos de conclusión de curso (TCC). Entre los problemas detectados se encuentran algunos relacionados directamente con elementos básicos 
de la investigación como: dificultad para la delimitación del tema, de los objetivos, la búsqueda del referencial teórico, dificultad con la elaboración de ideas, problemas a la hora de referenciar, conllevando a plagio en la mayoría de los casos.

Como resultado de su trabajo los especialistas antes mencionados llegan a presentarnos varias hipótesis:

1. La metodología de implementación de la asignatura tiene un carácter dual, trabajando la teoría separada de la práctica, lo que conlleva a un "aceptable" conocimiento teórico y serios problemas de aplicación de la práctica. Como defienden diferentes autores, no se puede aprender a investigar si no es investigando.

2. Dentro de la organización curricular de los cursos de graduación, la responsabilidad por aprender a investigar cae sobre una sola asignatura: Metodología de la Investigación, exonerando al resto de las asignaturas de la misión de formar un alumno/investigador. El componente investigación debe pasar a ser un eje transversal del currículo imperante.

3. Las dificultades de producción textual presentadas por los alumnos es el resultado de una mala base y poco desarrollo de hábitos y habilidades de lectura y escritura, arrastradas desde la educación básica, así como la ausencia de conocimientos básicos en el campo de la investigación, lo que conlleva a lecturas superficiales, comprometiendo el proceso de construcción de conocimientos.

4. Poco o casi ningún conocimiento por parte de los alumnos acerca de la importancia de la investigación en su preparación académica y profesional, provocada por la falta de contacto con los grupos de investigación presentes en la institución, los que funcionan solo para alumnos de la modalidad presencial. Los alumnos de la EAD no cuentan con programas específicos de iniciación científica, además de la precaria base con que llegan estos alumnos a la educación superior. 
La asignatura Metodología Científica, según nos presentan los autores ya mencionados, además de no tener una continuidad lógica en otras asignaturas del currículo del curso de Administración en EAD, no cuenta con apoyos externos de otros mecanismos que desarrollen la investigación como herramienta indispensable en la preparación de sus alumnos.

Estos problemas no son exclusivos del curso de Administración, vemos como en la mayoría de los cursos de licenciatura en EAD de la UFMA se repiten estos y otros problemas relacionados con los componentes de la investigación.

Otro de los temas que enfrentamos es lo relacionado con los contenidos y la forma de enseñar que deben ser abordados por la asignatura de Metodología Científica, ya que existen diferentes abordajes teóricos y modelos metodológicos, así como diversas opiniones de especialistas tanto dentro como fuera de Brasil.

Un tema recurrente está relacionado con la forma de enseñar la Metodología Científica en nuestras universidades. Este tema es poco abordado por la literatura especializada y resulta de gran interés a la hora de definir como enfrentar el desarrollo de la asignatura. Según el especialista mexicano Ricardo Sánchez (1990) encontramos tres formas de enseñar la investigación científica: la escolástica, la funcional y la practicista.

En el caso específico de la UFMA en la modalidad a distancia, vemos como la didáctica aplicada es un hibrido entre los dos primeros enfoques al enseñar la teoría, sus elementos estructurales y metodológicos y dejan fuera los elementos prácticos de cómo investigar, cómo realizar una investigación en la realidad y cómo incorporar la pesquisa como herramienta científica del día a día.

Según los estudios realizados podemos establecer que la gran mayoría de los autores consultados, incluyen los mismos temas, con algunas pocas diferencias, dadas a partir del perfil del curso, caracterizándose por ser, más bien, un manual de instrucciones de como realizar la investigación. Entre los temas que más se repiten se encuentran: estrategias de lectura, estudio y ciencia; qué es ciencia; trabajos científicos-investigación, tipos de investigación, redacción de trabajos científicos; proyecto de investigación, sus partes, etapas; métodos científicos; modalidad y metodologías 
de investigación; instrumentos para la recolección de datos; tratamiento a los datos; como utilizar las referencias bibliográficas (POLAK; DINIZ; SANTANA, 2014; RICHARDSON, 2014; SALOMON, 2010; MARCONI; LAKATOS, 2010; SEVERINO, 2007; LANTADA et al., 2003; RUIZ, 2002; LOBATO, 2001; SAMPIÉRI; COLLADO; LUCIO, 2013).

Se considera importante destacar que la gran mayoría de los estudios e investigaciones realizadas para determinar los contenidos básicos de la asignatura Metodología Científica, se refieren a la modalidad presencial y existen pocos trabajos dirigidos específicamente a la didáctica de metodología de investigación en EAD, lo que demuestra la necesidad de continuar trabajando e investigando para dar sustentaciones teórico-prácticas que enriquezcan las metodologías de trabajo en la EAD. Esto ha ocasionado que los programas de la asignatura Metodología Científica que se aplican en los cursos de licenciatura en EAD de la UFMA sean iguales o muy parecidos a los que se aplican en iguales cursos de la modalidad presencial, sin tener en cuenta algunas de las características ya expresadas que atañen específicamente a la EAD. Esto podría garantizar la organización didáctico-metodológica pensando en la innovación pedagógica del "Profesor Principal para EAD” (DOMINGO; MARTINS, 2017).

Otro de los problemas que se han detectado es el factor temas \& tiempo. Después de analizar la mayoría de los programas de Metodología Científica de los cursos de graduación a distancia de la UFMA (Administración, Lic. en Matemáticas, Lic. en Química, Lic. en Biología, Lic. en Artes, Lic. en Teatro y Lic. en Pedagogía) vimos que en su gran mayoría cuentan con 60 horas lectivas en su mapa curricular para intentar desarrollar los temas que relacionamos a continuación.

Temas o núcleos básicos de la asignatura Metodología de la Investigación:

- Ciencia y ciencia contemporánea;

- Papel de la metodología de la investigación;

- Producción textual;

- Conocimiento: tipos de conocimientos;

- Investigación: tipos de investigación (pura, fundamental, aplicada);

- Exploratoria, descriptiva, explicativa, bibliográfica, biográfica, 
documental, histórica, experimental, ex post facto, estudio de caso, estudio de campo, investigación acción;

- Investigación cualitativa y cuantitativa;

- Métodos de investigación: teóricos, empíricos, estadísticos, de expertos.

- Teóricos: inducción, deducción, hipotético, deductivo, comparativo, documental, modelación;

- Empíricos: observación, análisis documental, entrevista, encuesta, cuestionario, experimental, estudio de caso, estudio de campo;

- Estadísticos: descriptivo, inferencial;

- Métodos de expertos: criterios de expertos, método Delhfy;

- Proyecto de investigación, sus elementos y etapas;

- Presentación de resultados de investigación: elaboración de artículos, elaboración de monografías, elaboración de TCC;

- La ABNT (Asociación Brasileña de Normas Técnicas).

Con estos elementos se decidió aplicar una encuesta a un grupo de especialistas con el objetivo de conocer sus criterios sobre esta asignatura tanto de Brasil, como de otros países, con el fin de recibir sus opiniones sobre cuáles deberían ser los temas/núcleos a incluir en los programas de la asignatura Metodología Científica para los cursos de graduación en EAD.

De un total de 62 profesores consultados (por vía e-mail o presencialmente) el 100\% coincidió que estos deberían ser los temas a incluir en el programa de la asignatura.

Los especialistas consultados son todos profesores en el área de Metodología de la Investigación y con experiencia de trabajo de no menos de 3 años, la mayoría con experiencia también en EAD. Ellos son de la UFMA (21), de la UEMA (12), IFMA (8), Metodista de São Paulo (5), UNED (6), ICCP (6), otros (4).

Nos preguntamos: ¿Cómo podría ser realizada la magia de trabajar de manera apropiada todos estos temas en un espacio de 60 horas lectivas "a distancia"?

Consideramos oportuno comentar como en los cursos de la Facultad de Educación de la Universidad Nacional de Educación a Distancia (UNED) de España, casi estos mismos contenidos son impartidos en di- 
ferentes asignaturas y momentos del mapa curricular de los cursos de esta Facultad. Estas asignaturas son:

- Métodos de investigación en educación social (cód. 63012085);

- Métodos y diseño de Investigación en educación (cód. 63022095);

- Técnicas e instrumentos para la recogida de información (cód. 6302205);

- Investigación acción y sistematización de prácticas en educación social (Optativa).

Es importante resaltar que en el caso específico de la UNED, los alumnos de la Facultad de Educación reciben inicialmente la asignatura Estadística Aplicada a la Educación (Pérez Juste y otros, 2009) y para este fin cuentan con recursos audiovisuales, el AVA de la UNED y un material impreso (libro) que contiene los principales temas del programa de la asignatura, en el que los estudiantes pueden encontrar puntos de contacto con los contenidos del programa de Metodología de la Investigación, a saber:

- Problema de la investigación;

- Formulación de hipótesis y objetivos de investigación;

- Variables de hipótesis;

- Comprobación de objetivos;

- Elección del tema de investigación;

- Estructura y características del problema;

- Evaluación del problema;

- Fuentes y bases de documentación;

- Tratamiento de datos cuantitativos y cualitativos.

Consideramos importante destacar que la diferencia de los cursos de licenciatura a distancia de Brasil, en la UNED de España el paradigma cuantitativo/cualitativo es el imperante hasta la actualidad. Esto se demuestra en la carga de contenidos relacionados con la estadística aplicada a la investigación que reciben los discentes durante todo el período de sus estudios universitarios y también en los enfoques que presentan la gran mayoría de las investigaciones y trabajos finales de curso, realizado por los alumnos españoles, donde el diseño de investigación y el tratamiento de datos es fundamentalmente cuantitativo/cualitativo y no cualitativo como sucede actualmente en Brasil. 
La situación del post-grado en la Facultad de Educación de la UNED no es diferente a lo que se aprecia en los cursos de graduación. Encontramos que se presta una especial atención al tema de la investigación cuantitativa/cualitativa, lo que queda evidenciado en las diferentes asignaturas obligatorias y optativas que se encuentran en el plan de estudio que reciben los alumnos de los cursos de Maestría y Doctorado en esta Facultad.

Si por ejemplo, la Maestría de Cultura y Sociedad de la UFMA (PGCult) en su plan de estudios cuenta sólo con la asignatura de Seminario de Investigación I y II, cada uno con un total de 60 horas lectivas, donde el énfasis de los trabajos está dirigido a la investigación cualitativa, en los cursos de post-graduación de la Facultad de Educación de la UNED tienen:

- Diseños de investigación en educación (curso de nivelación para la entrada al máster) (cód. 23302028).

Este curso entre sus contenidos trabaja: Introducción a la investigación en educación, Diseños de investigación en educación y Medida en educación.

- Construcción y validación de Instrumentos para la recogida de datos (cód. 2330255);

- Innovación docente e iniciación a la investigación educativa (cód. 23304930);

- Investigación evaluativa (cód. 23302210).

En el caso específico del Máster Interuniversitario Euro-Latinoamericano en Educación Intercultural de la UNED, se han incluido asignaturas como:

- Investigación e interculturalidad en la sociedad del conocimiento (cód. 23305100);

- Metodología de investigación para el tratamiento educativo de la diversidad (cód. 23301415).

Como se puede apreciar a través de las asignaturas que se trabajan tanto en los cursos de graduación como en los cursos de post-graduación de la UNED, así como en la realización de trabajos de investigación, los alumnos que concluyen estos cursos, poseen amplios conocimientos en el campo de la investigación que los prepara efectivamente para enfrentarse con efectividad al mercado de trabajo y a la vida profesional. 
Podemos asegurar sin temor a equivocarnos, que de la manera que está organizada la actividad de investigación en los cursos de licenciados a distancia en la UFMA, basada en el desarrollo de una asignatura, sin otros apoyos internos o externos (pensar la investigación como herramienta de trabajo del resto de las asignaturas del currículo, becas de iniciación científica, grupos de investigación, entre otros), es extremadamente difícil desarrollar el gusto por la investigación en los alumnos y su incorporación como herramienta de trabajo en todo el proceso de su preparación como futuros cuadros científicos/técnicos.

Otro tema no menos importante es la utilización de recursos didácticos (medios de enseñanza-aprendizaje) para impartir la asignatura de Metodología Científica. Por um problema de espacio en este artículo recomendamos profundizar este aspecto en el trabajo publicado por este autor con el título de: Los objetos de aprendizaje (OA) para la asignatura de metodología de la investigación de los cursos de licenciatura a distancia. Disponível em: <http:// periodicos.unimesvirtual.com.br/index.php?journal=paideia $>$.

\section{Conclusiones}

Entre las responsabilidades de la universidad contemporánea está la de preparar a los futuros especialistas en el área de la investigación. Esta tiene que convertirse en una herramienta de trabajo para la resolución de problemas del cotidiano, por lo que todo esfuerzo que se haga en esta dirección está sumamente justificado.

La realidad actual es que los componentes de investigación que las universidades repasan para sus alumnos se centran en la asignatura de Metodología Cientifíca y en algunos casos en iniciativas de profesores como es el caso de los Grupos de Pesquisa y las Ligas académicas. Resulta interesante la propuesta de la Universidad Metodista de incluir los "Proyectos" como actividad conectada estrechamente con la pesquisa y actividades investigativas como eje transversal dentro de los cursos.

Existe poca literatura especializada sobre como desarrollar el componente "pesquisa" como eje transversal en los cursos de licenciatura de 
la modalidad a distancia. Se dan los primeros pasos en esta dirección y este artículo pretende incentivar la realización de investigaciones y actividades prácticas que tributen a este importante aspecto.

Vemos como perspectiva de futuro la sistematización de actividades y tareas de "investigación" durante todo el tiempo de los cursos de licenciatura como eje transversal usando la propuesta de Pedro Demo de actividades en tono mayor, medio y menor.

La inclusión de los grupos de pesquisa en los PAIED (con becas o sin ellas) es un camino importante al instaurar dentro de los cursos de licenciatura en la modalidad a distancia.

La definición de los contenidos básicos de la asignatura Metodología Científica, así como sus respectivos medios de enseñanza-aprendizaje será una de las líneas a seren trabajadas dentro de las universidades, inclusive pensando en la unificación de programas para la instauración de la figura del profesor principal como via de perfeccionamiento didáctico-metodológico de las asignaturas de los cursos de licenciatura.

\section{Referencias}

BAGNO, M. Pesquisa na Escola: o que é, como se faz. 23. ed. São Paulo: Loyola, 2009.

BRANDÃO, C. R. A Pergunta a Várias Mãos: A experiência da pesquisa no trabalho do educador. São Paulo: Cortez, 2003.

BISQUERRA, R. A. Metodología de la investigación educativa. Madrid: La Muralla, 2004.

CEREZAL M. J.; FIALlO R.J. Como Investigar en Pedagogía. Ciudad de La Habana: Pueblo y Educación, 2004.

CIAED. [Congressos Internacionais ABED de Educação a Distância: 2012, 2013, 2014, 2016, 2017]. Disponível em: <http://www.abed.org.br/hotsite/23ciaed/pt/apresentacao/>. Acesso em: 13 jan. 2018.

COLECTIVO DE AUTORES. Metodología de la investigación educacional: Desafíos y polémicas actuales. La Habana: Felix Varela, 2003. 
COUTINHO, C. P. Investigar on-line: desafios e oportunidades. In: BOTTENTUIT JÚNIOR, B. J.; COUTINHO, C. P. Educaçao on-line: Conceitos, metodologias, ferramentas e aplicaçoes. Curitiba: CRV, 2012. p. 9-27.

DEMO, P. Aprendizagem no Brasil: Ainda muito por fazer. Porto Alegre: Mediação, 2004a.

DEMO, P. Argumento de autoridade $\mathbf{X}$ autoridade do argumento: interfaces da cidadania e da epistemologia. Rio de Janeiro: Tempo Brasileiro, 2005.

DEMO, P. Complexidade e aprendizagem: a dinâmica não linear do conhecimento. São Paulo: Atlas, 2002.

DEMO, P. Conhecimento e aprendizagem na nova mídia. Brasília, DF: Plano, 2001.

DEMO, P. Educação e qualidade. Campinas: Papirus, 1994.

DEMO, P. Educar pela pesquisa. 10. ed. Campinas: Autores Associados, 2015.

DEMO, P. Formação Permanente e Tecnologias Educacionais. Petrópolis: Vozes, 2006.

DEMO, P. Metodologia do Conhecimento Científico. São Paulo: Atlas, 2000a.

DEMO, P. O Porvir: Novas linguagens do século XXI. Curitiba: IBPEX, 2007.

DEMO, P. Pesquisa e construção de conhecimento: metodologia científica no caminho de Habermas. Rio de Janeiro: Tempo Brasileiro, 2009.

DEMO, P. Pesquisa Participante: Saber pensar e intervir juntos. Brasília, DF: LiberLivro, 2004b.

DEMO, P. Saber Pensar. São Paulo: Cortez, 2000b.

DEMO, P. Saber pensar. 6. ed. São Paulo: Cortez/Instituto Paulo Freire, 2008a.

DEMO, P. TICs e educação. 2008b. Disponível em: < http://pedrodemo.blogspot.com.es/2012/04/tics-e-educacao.html>. Acesso em: 10 out. 2010.

DOMINGO, R. P. Los objetos de aprendizaje (OA) para la asignatura de metodologia de la investigacion de los cursos de licenciatura a distancia. 2018. 
Disponível em: <http://periodicos.unimesvirtual.com.br/index.php?journal=paideia>. Acesso em: 13 jan. 2018.

DOMINGO, R. P.; MARTINS M. F. Profesor Principal para EAD. Trabalho apresentado no $23^{\circ}$ Congresso Internacional ABED de Educação a Distância, Foz do Iguaçu, 2017. Disponível em: < http://www.abed.org.br/congresso2017/ trabalhos/pdf/109.pdf>. Acesso em: 13 jan. 2018.

DOMINGO, R. P. Sistema de Medios de enseñanza para el desarrollo de hábitos y habilidades de lectura en los IPVCE de la República de Cuba. 1992. 188 f. Tesis (Doctorado) - Academia de Educacion de Rusia, Moscu, 1992.

GALAN, G. A. La investigación cuantitativa en educación especial. In: GONZÁLES PÉREZ, J. (Coord.). Necesidades educativas especiales e intervención psicopedagógica. Madrid: Universidad de Alcalá, 2003.

GALAN, A. Incertidumbres y desafíos de la universidad española. Escenarios posibles a corto y medio plazo. Avances en supervisión educativa, n. 19, nov. 2013.

GALIAZZI, M. do C.; MORAES, R. Educação pela pesquisa como modo, tempo e espaço de qualificação da formação de professores de ciências. Ciência $\boldsymbol{\&}$ Educação, Bauru, v. 8, n. 2, p. 237-252, 2002.

GARCÍA-LLAMAS, J. L. et al. Introducción a la investigación en educación. Madrid: UNED, 2003.

LANTADA, M. M. et al. (Org.). Metodología de la investigación educacional: desafíos y polémicas actuales. Ciudad de La Habana, Cuba: Editorial Félix Varela, 2003.

LOBATO, R. M. Metodologia da pesquisa. São Luís: Edições UFMA, 2001.

MARCONI, M. de A.; LAKATOS, E. M. Fundamentos de metodologia científica. 7. ed. São Paulo: Atlas, 2010.

MARQUES, M. O. Escrever é Preciso: O princípio da pesquisa. Ijuí: Unijui, 1997.

MORAES, R.; LIMA, V. M. R. (Org.). Pesquisa em Sala de Aula: Tendências para a educação em novos tempos. Porto Alegre: EDIPUCRS, 2002.

MORIN, E. Introdução ao Pensamento Complexo. Lisboa: Instituto Piaget, 1995. 
MORIN, E. La Méthode - 5. L'humanité de l'humanité. L'identité humaine. Paris: Seuil, 2002.

OLIVEIRA, L. C. de; OLIVEIRA JÚNIOR, H. R. de. A pesquisa na educação a distância: uma análise acerca da asignatura metodologia da pesquisa no curso de Administração da UFMA. In: ESUD 2012 - CONGRESSO BRASILEIRO DE ENSINO SUPERIOR A DISTÂNCIA, 9. Educação a Distância: Semeando Cidadania, 2012, Recife. Anais... Recife: UniRede, 2012. p. 1-16.

OLIVEIRA, L. C. Esquina da pesquisa: uma proposta para educar pela pesquisa os alunos dos cursos de licenciatura em EAD da UFMA. 2015. Dissertação (Mestrado) - Universidade Federal do Maranhão, São Luís, 2015.

PEREZ JUSTE, R. Evaluación de programas educativos. Madrid: La Muralla, 2006.

PEREZ JUSTE, R.; GALAN, G. A.; QUINTANAL, D. J. Métodos y Diseños de Investigación en Educación. Madrid: UNED, 2012.

PEREZ JUSTE, R. et al. Estadística aplicada a la educación. Madrid: Pearson Educación, 2009.

PEREZ JUSTE, R. Métodos cualitativos en la investigación en Educación a Distancia, tema 62. Madrid: UNED, 2011. (Edición no venal).

PRESTES, M. L. M. A Pesquisa e a Construção do Conhecimento Científico: Do planejamento aos textos, da escola à academia. São Paulo: Respel, 2003.

POLAK, Y. N. de S.; DINIZ, J. A.; SANTANA, J. R. Dialogando sobre Metodología Científica. Fortaleza: UFC, 2014.

RICHARDSON, R. J. (Org.). Pesquisa social: métodos e técnicas. 3. ed. rev. e atual. São Paulo: Atlas, 2014.

RUIZ, A. A. La investigación en la educación. Una Introducción a la investigación en la educación. Sto Domingo: Editorial Aplusele, 2007.

RUIZ, J. Á. Metodologia científica: guia para eficiência nos estudos. 5. ed. São Paulo: Atlas, 2002.

SALOMON, D. V. Como fazer uma monografia. 12. ed. São Paulo: Martins Fontes, 2010. 
SAMPIÉRI, R. H.; COLLADO, C. F.; LUCIO, P. B. Metodologia de pesquisa. 5. ed. Porto Alegre: Penso, 2013.

SANCHEZ, R. Didáctica de la metodología de la investigación. México, D.F., 1990.

SEVERINO, A. J. Metodologia do trabalho científico. 23. ed. rev. e atual. São Paulo: Cortez, 2007.

SILVA, T. M. N. A construção do currículo na sala de aula: o professor como pesquisador. São Paulo: EPU, 1990.

Submetido em: 9-5-2018

Aceito em: 29-5-2018 